\title{
Exporting Vector Muscles for Facial Animation
}

\author{
The Duy Bui, Dirk Heylen, Mannes Poel, and Anton Nijholt \\ University of Twente \\ Department of Computer Science \\ The Netherlands \\ \{theduy, heylen, mpoel, anijholt\}@cs.utwente.nl
}

\begin{abstract}
In this paper we introduce a method of exporting vector muscles from one 3D face to another for facial animation. Starting from a 3D face with an extended version of Waters' linear muscle system, we transfer the linear muscles to a target $3 \mathrm{D}$ face. We also transfer the region division, which is used to increase the performance of the muscle as well as to control the animation. The human involvement is just as simple as selecting the faces which shows the most natural facial expressions in the animator's view. The method allows the transfer of the animation to a new 3D model within a short time. The transferred muscles can then be used to create new animations.
\end{abstract}

\section{Introduction}

Facial animation aims at producing realistic facial expression to enrich human computer interaction. Various techniques have been introduced to animate the 3D faces, including: interpolation ([18]), parameterizations ([6]), finite element methods ([12], [13]), pseudo muscle models ([11]) and physics based muscle models ([9], [20], [21]). One of the issues related to these techniques is how to transfer the animation parameters to a newly created face model.

Noh and Neumann [17] have proposed a method for transferring animation between different head models on the geometric level. Surface correspondences are obtained by Radial Basis Function mapping using the specification of corresponding point pairs on the models. Some heuristics for feature detection are presented as well to reduce the number of feature points which have to be selected manually. The detected feature points also need to be adjusted manually afterwards to achieve more correct mapping. Moreover, this method can only transfer pre-created animations. No new animation can be created in the new face. Kähler et al. [10] transfer both the head model and the underlying muscle and bone structure to a new face based on the interpolation of two sets of landmarks. The method still requires the manual adjustment on the sets of landmarks by point-and-click. They claim that it takes 10-20 minutes to specify the 60 needed landmarks. Mani and Ostermann [14] use B-splines with weights to clone MPEG-4 facial animation tables (FAT) from a source face model to a target face model. Manual selection of corresponding points between source and target faces is required. Additional manual adjustment of B-splines weights is also required to increase the correctness in mapping the MPEG-4 FATs, however this correctness is still not warranted. 
In this paper we introduce a method of exporting vector muscles from one face to another face for facial animation. We have built a system to convert from emotion intensities to muscle contraction levels [3] to display emotional facial expression in a 3D face. We certainly do not want to adjust the muscle structure manually on a new face in order to reuse these muscle contraction levels. Starting from a 3D face with an extended version of Waters' linear muscle system (discussed in Section 2), we transfer the linear muscles to a target 3D face. In the extended version, the face is also divided into regions that are used to increase the performance of the muscle as well as to control the animation.

Muscle contraction values are transferable between models only when the models involved are equipped with properly positioned muscles. Even then, a problem arises when muscle structures or surface shapes are inherently different between two models. The system we describe in this paper allows the transfer of muscle between 3D face models with minimal human involvement. The human contribution is just as simple as selecting the "favored" version of different expressions on the face. The transferred muscles can then be used to create new animations. The transfer of the muscles is first done by mapping the muscles to the target 3D face with Radial Basis Functions mapping. This mapping produces the rough version of the muscles on the target $3 \mathrm{D}$ face with the information from an existing muscle system. The muscles are then tuned up in an interactive process. This process uses Interactive Genetic Algorithms as an underlying technique. This procedure is explained in Section 3. The region division is transferred by Radial Basis Functions mapping followed by "cylindrical projections". More details are provided in Section 4

\section{The Vector Muscle Model}

The vector muscle model was introduced by Waters [21] to create realistic facial expressions in realtime. The muscle's zone of influence are illustrated in Figure 1. The muscle is modelled as a vector in 3D space, from $v_{2}$ to $v_{1} . R_{s}$ and $R_{f}$ represent the fall-off radius start and finish respectively. Under the muscle's contraction, the new vertex $p^{\prime}$ of an arbitrary vertex $p$ located on the mesh within the segment $v_{1} p_{r} p_{s}$, along the vector $p-v_{1}$, is computed as follows:

$$
p^{\prime}=p+\cos (\alpha) k r \frac{p-v_{1}}{\left\|p-v_{1}\right\|}
$$

where $\alpha$ is the angle between the vector $v_{2}-v_{1}$ and $p-v_{1}, D$ is $\left\|p-v_{1}\right\|, k$ is a fixed constant representing the elasticity of skin, and $r$ is the radial displacement parameter:

$$
r= \begin{cases}\cos \left(1-\frac{D}{R_{s}}\right) & \text { for } \mathrm{p} \text { inside sector }\left(v_{1} p_{n} p_{m}\right) \\ \cos \left(\frac{D-R_{s}}{R_{f}-R_{s}}\right) & \text { for } \mathrm{p} \text { inside sector }\left(p_{n} p_{r} p_{s} p_{m}\right)\end{cases}
$$

We have extended this model to deal with multiple muscles interaction, to increase the performance and to produce realistic wrinkles [4]. We simulated the parallelism in the way multiple facial muscles contract. How two muscles interact can be seen in Figure 2(a) Using region division on the face (see Figure 7), the calculation time for the muscle model is significantly reduced. We also use parabola to simulate wrinkles for this muscle model. An example of the wrinkles on the forehead is shown in Figure 2(b).

Exporting a muscle system from the source face to the target face means that we have to find the positions of the head and tail of the muscles in the target face based on 


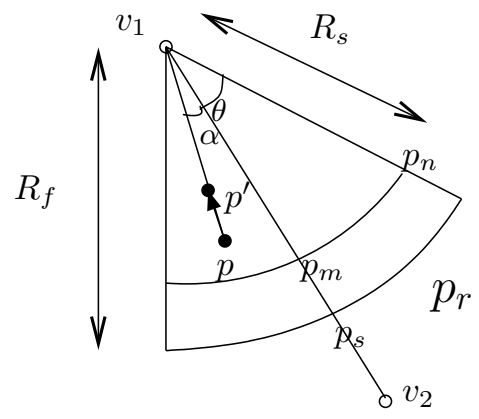

Fig. 1. The linear muscle model

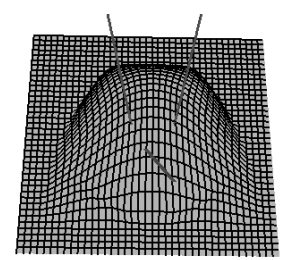

(a)

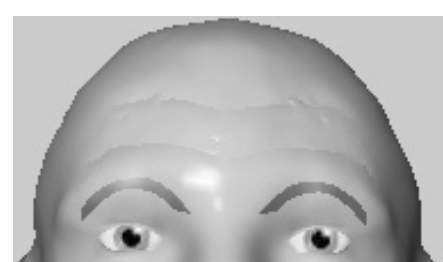

(b)

Fig. 2. Improvements on Waters' vector muscle model: (a) how two muscles interact; (b) wrinkles on the forehead

their positions in the source face. Other parameters such as the values of fall-in start and fall-in finish are also transferred and adjusted.

\section{Transferring the Muscles}

The muscles are transferred to the new face interactively. First the muscles are interpolated to the new face using Radial Basis Functions (RBF). The RBFs use several pairs of corresponding feature points to establish a rough version of the transferred muscles. Groups of muscles are then further tuned in an interactive process using Interactive $\mathrm{Ge}$ netic Algorithms as the underlying technique. Several versions of the same expression with different versions of these muscles are repeatedly shown to animator. The animator selects the faces which show the most natural expression from the animator's point of view. This selection process finishes when the animator is satisfied with the facial expression performance of the face. The positions of original muscles on the source face and the tuned muscles are added to the set of corresponding feature points. The muscles are interpolated again with the new set of correspondence and a new group of muscles are tuned. This procedure is performed six times for six different groups of muscles. These six groups of muscles are the muscles used to produce versions of six basic expressions: happiness, sadness, surprise, anger, fear and disgust. 
The algorithm can be described as follows. Let $L_{\text {source }}$ denote the set of feature points of the source face, $L_{\text {target }}$ the set of feature points of the target face, $M_{\text {source }}$ the set of vertices represent the muscles (head and tail of the muscle) in the source face, and $M_{\text {target }}$ the set of vertices represent the muscles (head and tail of the muscle) in the target face. The steps to transfer the muscles is described as follows:

For expression E=Happiness,Surprise,Sadness,Anger,Fear,Disgust

RBF_mapping $\left(M_{\text {source }}, L_{\text {source }}\right.$, Ltarget $) \rightarrow M_{\text {target }}$

Repeat (Interactive Genetic Algorithm)

Show different versions of expression $E$

The user chooses some "favored" faces

The system calculates new faces

Until the user satisfies with the faces

Add head and tail of the muscles that express $E$ to $L_{\text {source }}$ and $L_{\text {target }}$

Note that the order of the expressions is from the easier recognized ones to more difficult recognized ones. We will now discuss in more detail the Radial Basis Functions and the Interactive Genetic Algorithms.

\subsection{Radial Basis Functions}

The initial muscle transformation from the source face to the target face is done by Radial Basis Function (RBF) Networks. These are known for their powerful interpolation capability and are often used for face model fitting [17].

For each coordinate a different RBF network is defined, the transformation has the form

$$
(x, y, z) \longrightarrow\left(R B F_{1}(x, y, z), R B F_{2}(x, y, z), R B F_{3}(x, y, z)\right)
$$

where each $R B F_{i}$ is given by

$$
R B F_{i}(x, y, z)=\sum_{j=1}^{n} w_{i, j} h_{i, j}(x, y, z)
$$

where the $w_{i, j}$ are weights of the network and need to be determined or learned on the basis of a training set. For the basis functions $h_{i, j}$ we follow the successful approach given in [17]:

$$
h_{i, j}(v)=\sqrt{\left\|v-\mu_{i, j}\right\|^{2}+s_{i, j}^{2}}
$$

where $\mu_{i, j}$ is the center and $s_{i, j}$ is given by

$$
s_{i, j}=\min _{k \neq j}\left\|\mu_{i, k}-\mu_{i, j}\right\|
$$

This choice for $s_{i, j}$ leads to smaller deformations for widely scattered center points and larger deformations for closely located points.

Each of the RBF networks is trained using a set of coordinates of feature points on the source face and the corresponding feature points on the target face. These feature points are: 
- Tip of the nose

- Top of the head

- Right side of the face

- Left side of the face

- Top of the nose (between two eyes)

- Left eye socket (near nose)

- Right eye socket (near nose)

- Bottom of the nose (top of the furrow)

- Bottom left of the nose

- Bottom right of the nose

These feature points can be determined automatically, c.f. [17].

Each time a group of muscles is positioned at the right place, these muscle coordinates are added to features set. Afterwards the RBF network is trained again in order to interpolate more accurately. And in order to prevent overfitting and to improve generalization a regularization term $\sum_{j} w_{i, j}^{2}$ is added to the error term for each $R B F_{i}$ [2]

\subsection{Muscle Tuning}

A rough version of the muscles is produced from the RBF mapping. Note that, the RBF mapping only transfers the position of the muscles. Other parameters such as the influence angle $\alpha$, are simply copied from the source muscles. The position of the muscles and other parameters still need to be adjusted to be usable on the target face. We use an Interactive Genetic Algorithms (IGA) system to further adjust the muscles.

The Interactive Genetic Algorithms. Genetic Algorithms are search algorithms based on the process of natural evolution [8]. A Genetic Algorithm's problem solving approach is, basically, to rate or to rank possible solutions as they are generated, and then use these rankings to guide further exploration for related solutions. For a more thorough introduction of Genetic Algorithms, the readers can consult [8] and [7].

While conventional Genetic Algorithms use external determined fitness functions, Interactive Genetic Algorithms are guided by the user's intuition preference, subjectivity,sensation, perception, cognition and other psychological processing functions [19]. Instead of rating chromosomes on certain values (fitness), Interactive Genetic Algorithms rate chromosomes interactively based on evaluations of a user. Interactive Genetic Algorithms are useful for searching through an object space when assessment criteria are not well defined enough to form explicit rules [16]. Some applications of Genetic Algorithms are interactive design aid system [15], montage face image generation [5] and line drawing [1].

Using IGA for tuning up muscles. A linear muscle can be described as a set of ten parameters, $v_{1}, v_{2}, \ldots, v_{10}$ :

- coordinates of the head of the muscle (3 parameters)

- coordinates of the tail of the muscle (3 parameters) 


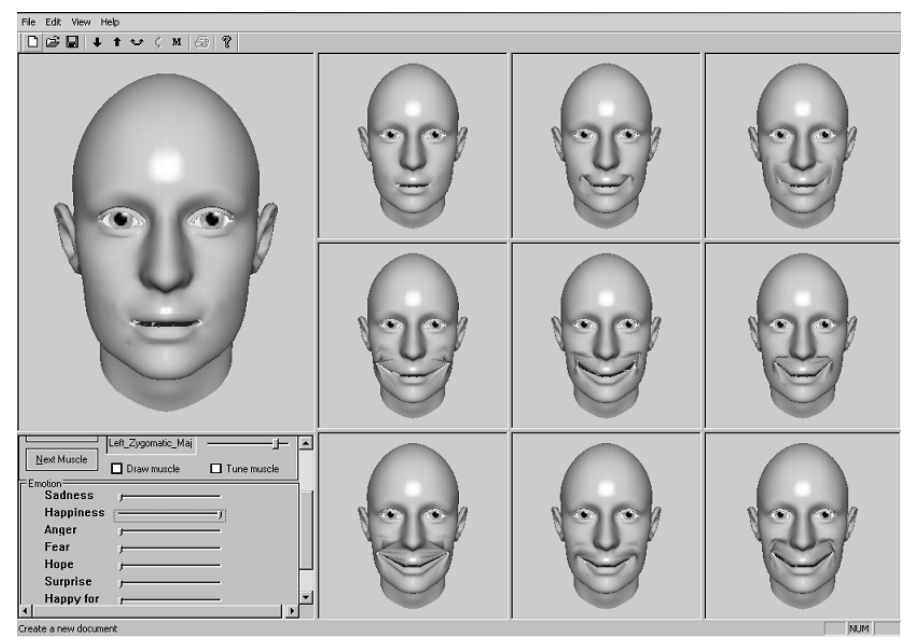

Fig. 3. A snapshot of IGA muscle tuning system

- the value of fall-off start

- the value of fall-off finish

- the value of the angle

- the value of the standard to transform contraction level to $(0 . .1)$

Starting from an initial version of a muscle, numbers of versions of the muscle are created by modifying each parameter in a specified range. Let $R \min _{1}, \operatorname{Rmax}_{1}, \ldots, \operatorname{Rmin}_{10}$, $R \max _{10}$ be the ranges of values of the parameters. The parameters of a muscle can be represented as:

$$
\left(p_{1}, p_{2}, \ldots, p_{10}\right)
$$

where $0.0 \leq p_{i} \leq 1.0$

$$
\begin{aligned}
p_{i} & =\frac{v_{i}-\operatorname{Rmin}_{i}}{\operatorname{Rmax}_{i}-\operatorname{Rmin}_{i}}, \\
v_{i} & =p_{i}\left(\operatorname{Rmax}_{i}-\operatorname{Rmin}_{i}\right)+\operatorname{Rmin}_{i} \quad i=1 . .10
\end{aligned}
$$

A chromosome representing a muscle looks like:

$$
c_{11}, c_{12}, \ldots, c_{1 n}, c_{21}, \ldots, c_{10 n} \quad c_{i j}=0 \text { or } 1
$$

where $2^{n}$ is used to convert a real number between 0.0 and 1.0 to a binary string. That means $c_{i 1}, c_{i 2}, \ldots, c_{i n}$ is the binary representation of $\left\lfloor 2^{n} p_{i}\right\rfloor$

The muscles are now ready to be tuned with the IGA. At each iteration of IGA, nine faces with the same expression but different versions of the muscles are shown to the animator. The animator selects the three faces that best represent the specified expression. Based on the three selected faces, a new generation of versions of muscles is generated. The faces which are contracted with these versions of muscles are shown to the animator again. This process finishes when the animator find a satisfactory version of the muscles. A snapshot of the system is shown in Figure 3. 


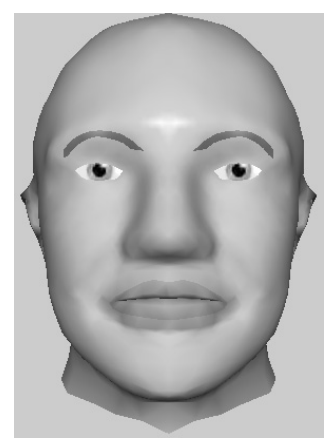

(a)

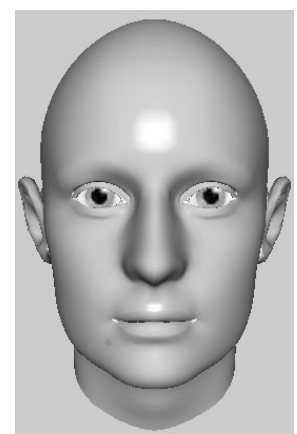

(b)

Fig. 4. (a) The source face; (b) the target face

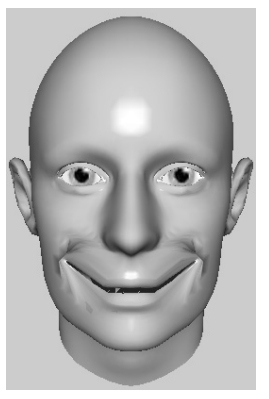

(a)

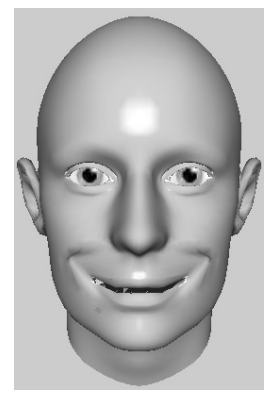

(b)

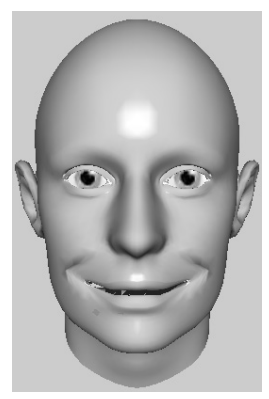

(c)

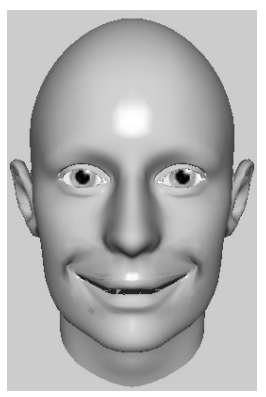

(d)

Fig. 5. Tuning up the muscles for happiness: (a) - result from RBF mapping; (b),(c),(d) - step by step result from IGA tuning system

\subsection{Result}

An example of a source face and a target face can be seen in Figure 4 We will now show the result of exporting muscles that create happiness and sadness. The rough version of the muscles that produce happiness is shown in Figure 5(a). As can be seen from that Figure, RBF mapping does not immediately generate a usable version of the muscles. The muscles are then interactively adjusted with the IGA system. Figure 5(b) and 5(c) present the intermediate versions of the muscles during the IGA process. Figure 5(d) shows the final adjusted result. How the muscles that create sadness are transferred is shown in Figure 6 Muscles that create other expressions are transferred in the same manner. 


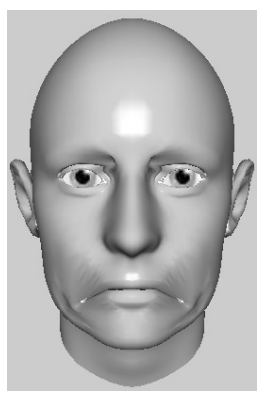

(a)

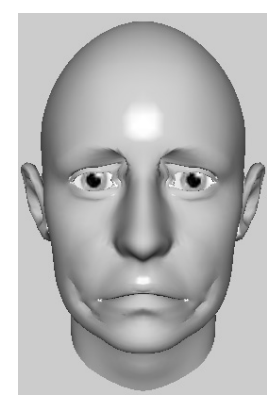

(b)

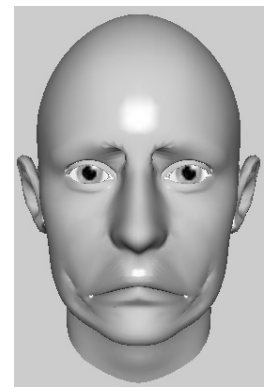

(c)

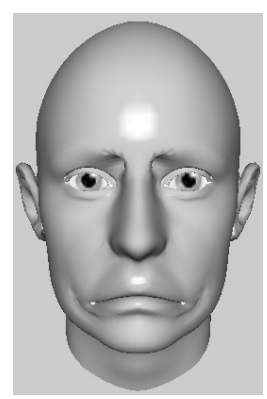

(d)

Fig. 6. Tuning up the muscles for sadness: (a) - result from RBF mapping; (b),(c),(d) - step by step result from IGA tuning system

\section{Transferring Region Division to the New 3D Face}

Region division has been used to accelerate the calculation of the linear muscle in realtime [4]. The region division is also used by the muscles to control animation, e.g. the lower-face muscles do not effect the upper lip. For this reason, we also have to transfer the region division to the new face. This time, we map the source face to the target face with RBF and the set of correspondences which are described above. We then use "cylindrical projections" [17] to project the target face into the mapped version of the source face. This works as follows. Each vertex of the target $3 \mathrm{D}$ face $v$ is projected into a triangle $\left(v_{1}, v_{2}, v_{3}\right)$ on the mapped version of the source face. The region that vertex $v$ belongs to will be the region of one of the vertices $\left(v_{1}, v_{2}, v_{3}\right)$. In this case, we take the vertex $v_{i}$ with shortest distance to the vertex $v$.

Special care has to be paid to the lips. Only RBF mapping followed by "cylindrical projections" does not produce correct division between the upper and the lower lips. A further step has to be taken in order to solve this problem. We search from the tip of the nose down to the chin, find all the vertices that are upper-opened 1 (see Figure 8(b) . These vertices form the contact lines of the lower lip. We expand down from these vertices to the chin and mark all the vertices in between to be in the lower face region. We then search for all the vertices that are lower-opened (see Figure 8(a). Expanding up from these vertices to the tip of the nose, we mark all the vertices in between to be in the middle face region.

The regions division in the target face can be seen in Figure 7(b).

\footnotetext{
${ }^{1} \mathrm{~A}$ vertex is upper-opened if the intersections between all polygons containing the vertex and the line that goes through the vertex and is parallel with Y-axis "lie under" the vertex.

${ }^{2} \mathrm{~A}$ vertex is lower-opened if the intersections between all polygons containing the vertex and the line that goes through the vertex and is parallel with Y-axis "lie above" the vertex.
} 


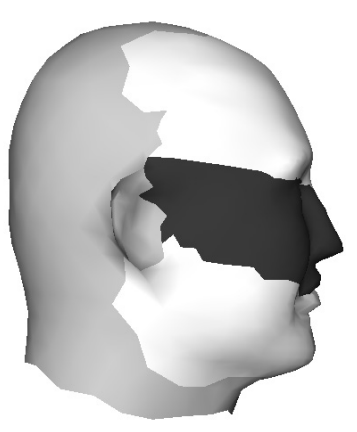

(a)

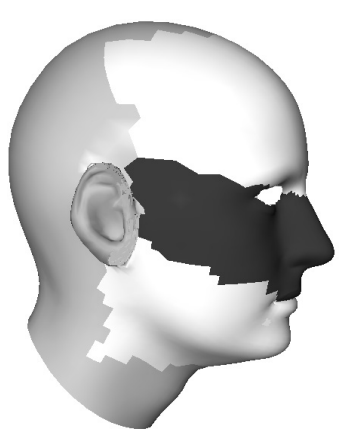

(b)

Fig. 7. The transfer of region division: (a) the region division on the source face; (b) the region division on the target face

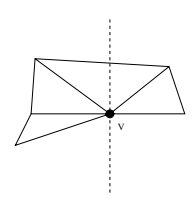

(a)

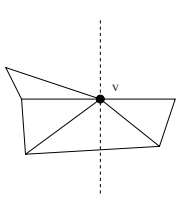

(b)

Fig. 8. Example of (a) lower-opened vertex; (b) upper-opened vertex

\section{Conclusion}

We have introduced a method of exporting linear muscles from one 3D face to another. The transfer of the muscles was first done by mapping the muscles to the target 3D face with Radial Basis Functions mapping. This mapping produced the rough version of the muscles on the target 3D face with the information from an existing muscle system. The muscles are then tuned up by an IGA system. We have also shown how to transfer the region division. The system produces natural facial expression when all the muscles are adjusted. The whole process takes about 10-20 minutes. The main advantage of this system is that the animator only has to select the expressed face instead of manually tuning the muscle parameters.

\section{References}

1. Baker, E. \& M., Seltzer (1994), "Evolving line drawings", In: Graphics Interface'94 Proc., Banff, Alberta, Canada, edited by Wayne A, Davis \& Barry Joe. Morgan Kaufmann Publishers, pp. 91-100.

2. Bishop,C.M. (1995), Neural Networks for Pattern Recognition, Clarendon Press-Oxford. 
3. Bui,T.D., Heylen,D., Poel, M. and Nijholt, A. (2001), "Generation of facial expressions from emotion using a fuzzy rule based system", In: Lecture Notes in Artificial Intelligence 2256, M. Stumptner, D. Corbett \& M. Brooks (eds.), Springer, Berlin, 83-94.

4. Bui, T.D., D. Heylen \& A. Nijholt (2003), "Improvements on a simple muscle-based 3D face for realistic facial expressions", In: 16th International Conference on Computer Animation and Social Agents (CASA'2003), Rutgers University, May 2003, to appear.

5. Caldwell, C. \& V.S., Johnston (1991), "Tracking of criminal suspect through face-space with a genetic algorithm", In: 4th International Conference on Genetic Algorithms (ICGA'91), San Diego, CA, USA, pp. 416-421.

6. Cohen, M. M., \& Massaro, D. W. (1993), "Modeling coarticulation in synthetic visual speech", In N. M. Thalmann \& D. Thalmann (Eds.) Models and Techniques in Computer Animation, Tokyo: Springer-Verlag.

7. Davis, L. (ed.) (1991). Handbook of Genetic Algorithms, Van Nostrand Reinhold.

8. Goldberg, D.E. (1989), Genetic Algorithms in Search, Optimization, and Machine Learning, Addison-Wesley Publishing Company Inc.

9. Kähler, K., J. Haber and H.-P. Seidel (2001), "Geometry-based muscle modeling for facial animation", Proceedings Graphics Interface 2001, pp. 37-46.

10. Kähler, K., Haber, J., Yamauchi, H., Seidel, H., (2002), "Head Shop: Generating animated head models with anatomical structure", ACM SIGGRAPH Symposium on Computer Animation, pp. 55-64.

11. Kalra P, Mangili A, Magnenat-Thalmann N, Thalmann D (1992), "Simulation of Facial Muscle Actions Based on Rational Free Form Deformations", Proc. Eurographics '92, Cambridge, U.K., Computer Graphics Forum, Vol. 2, No. 3, pp. 59-69.

12. Rolf M. Koch, Markus H. Gross, Friedrich R. Carls, Daniel F. von Büren, Yoav I. H. Parish (1996), "Simulating Facial Surgery Using Finite Element Models", In Computer Graphics Proceedings, Annual Conference Series, ACM SIGGRAPH, pp. 421-428.

13. Rolf M. Koch, Markus H. Gross, Albert Bosshard (1998), "Emotion Editing using Finite Elements", Computer Graphics Forum 17(3): 295-302.

14. M. V. Mani, J. Ostermann, "Cloning of MPEG-4 face models", International Workshop on Very Low Bit rate Video Coding (VLBV 01), Athens, October, 2001.

15. Nakanishi, Y. (1996), "Applying Evolutionary Systems to Design Aid System", In: ALIFEV, Poster Presentation, PP-25, pp. 147-154.

16. Nishio, K., Murakami, M., Mizutani, E. \& N., Honda (1997), "Fuzzy Fitness Assignment in an Interactive Genetic Algorithm for a Cartoon Face Search", In: Genetic Algorithm and Fuzzy Logic Systems, Sanchez, E. et al (eds), World Scientific Publishing Co., Singapore.

17. J.Y. Noh and U. Neumann (2001), "Expression Cloning", Computer Graphics, Proceedings of ACM SIGGRAPH 2001, Los Angeles CA, pp. 277-288.

18. Parke, F. I. (1991), "Techniques for facial animation", In N. Magnenat-Thalmann and D. Thalmann (Eds.), New Trends in Animation and Visualization, John Wiley, Chichester, 229241.

19. Takagi, H. (1998), "Interactive evolutionary computation: Cooperation of computational intelligent and human kansei", In: Proceeding of 5th International conference on Soft Computing, pp. 41-50, Iizuka, Japan.

20. Terzopoulos, D. and Waters, K. (1990), "Physically-Based Facial Modeling, Analysis, and Animation", The Journal of Visualization and Computer Animation, Vol.1, pages 73-80.

21. Waters, K. (1987), "A muscle model for animating three-dimensional facial expressions", Computer Graphics (SIGGRAPH'87), 21(4), 17-24. 